\title{
Psychiatric Consultations at an Emergency Department in a Metropolitan University Hospital in Northern Japan
}

\author{
Masaki Shiraishi', Takao Ishii', Yoshiyasu Kigawa', Masaya Tayama', Keisuke Inoue², \\ Kenji Narita', Masaru Tateno', and Chiaki Kawanishi' ${ }^{1}{ }^{\circledR}$ \\ 1Department of Neuropsychiatry, Sapporo Medical University Graduate School of Medicine, Sapporo, Japan \\ ${ }^{2}$ Psychiatric Center, Yokohama City University Medical Center, Yokohama, Japan
}

Many patients with mental disorders visit emergency departments (EDs). However, the majority of these patients do not receive psychiatric assessment. In the present study, we investigated the detailed proportion of patients with mental disorders visiting an urban ED in the largest northern city in Japan. A retrospective chart review study was performed at a University Hospital from January 2012 to December 2015. The reasons for psychiatric consultations made by ED staff, and the primary psychiatric diagnoses were investigated. Among all living patients, $20 \%$ of them received consultations. The most common reason for consultation was suicide attempt followed by agitation or insomnia. Of all diagnoses, organic mental disorder was the most frequent and the mean age was significantly higher than the other diagnostic groups. Our study indicated that the frequency of psychiatric consultation was high. This indicates the high demand for mental health services at the ED. A thorough psychiatric assessment can provide adequate psychiatric services to acute patients; thereby possibly preventing suicide attempters from later actually dying by suicide.

Psychiatry Investig 2018;15(7):739-742

Key Words Emergency department, Psychiatric assessment, Psychiatric consultation, Psychiatric emergency, Suicide.

\section{INTRODUCTION}

Many patients with mental disorders or suicide-related behaviors visit emergency departments (EDs). In 2005, Larkin analyzed 53 million mental health-related visits between 1992 and 2001, reporting an increase from 4.9\% to 6.3\% for all EDs during that decade. ${ }^{1}$ Of all visits to EDs in a primary study conducted in Australia, the frequency of mental illness visits was reported to range from $2.4 \%$ to $6.6 \%{ }^{2}$ Additionally, a systematic review reported that the proportion of all ED episodes due to mental illness was $4 \%$, and a third was due to deliberate self-harm, suicide attempt, or suicidal ideation. ${ }^{3}$ In Japan, mental health-related ED visits have been reported to range from $3.6 \%$ to $5.6 \%$ in the last decade according to the

Received: January 4, 2018 Revised: March 6, 2018

Accepted: April 4, 2018

$\triangle$ Correspondence: Chiaki Kawanishi, MD, PhD

Department of Neuropsychiatry, Sapporo Medical University Graduate School of Medicine, S-1, W-16, Chuo-ku, Sapporo 060-8543, Japan

Tel: +81-11-611-2111, Fax: +81-11-644-3041,

E-mail: chiaki.kawanishi@gmail.com

(ㄷ) This is an Open Access article distributed under the terms of the Creative Commons Attribution Non-Commercial License (http://creativecommons.org/licenses/by$\mathrm{nc} / 4.0$ ) which permits unrestricted non-commercial use, distribution, and reproduction in any medium, provided the original work is properly cited. annual reports of the Fire and Disaster Management Agency. ${ }^{4}$ However, Nishi et al. ${ }^{5}$ reported that the percentage of psychiatric-related ED visits was very high (14.4\%) in an emergency center in Tokyo.

The majority of ED patients with mental disorders do not receive assessment regarding their mental health conditions or psychiatric symptoms at this opportunistic point of contact. ${ }^{6,7}$ In the United Kingdom, 58.9\% of all deliberate selfharm patients did not receive a psychiatric assessment. ${ }^{8}$

In Asian countries, systematic reviews focused on suicidal behavior have reported on the proportion of suicide attempters in EDs. ${ }^{9}$ However, few studies have explored the detailed proportion of patients with mental disorders visiting EDs.

In the present study, we investigated the detailed proportion of patients with mental disorders visiting an urban ED in the largest northern city in Japan. We examined reasons for psychiatric consultations made by ED staff, and examined psychiatric diagnoses for each patient who received consultation.

\section{METHODS}

A retrospective chart review study was performed. Patients 
who were admitted to the Advanced Critical Care and Emergency Center (ACCEC) of Sapporo Medical University Hospital between January 2012 and December 2015 were targeted. Sapporo Medical University Hospital and its ACCEC are located in the center of Sapporo, the fifth most populated city in Japan (1.9 million). The ACCEC is a special ED initiated by the Ministry of Health, Labour and Welfare, Japan, which provides a 24-hour medical care system for treating extensive burns, dismemberment, acute poisoning, and other severe cases in the prefecture. Regarding suicide-related behaviors, only patients with a high risk of mortality are sent to the special ED. Japan has 38 special EDs; ACCEC in Sapporo Medical University Hospital is the only special ED serving the entire population of Hokkaido Prefecture, the geographically largest prefecture in Japan (population 5.3 million).

Among all targeted patients, we recruited those who received consultation at the psychiatric department. As a general rule, psychiatric consultations are booked by ED staff on a medical record ordering system if necessary at the ACCEC. In addition to basic demographic data, we collected the reasons for each consultation, as recorded by ED staff at the ACCEC. The reasons for consultation were classified into five categories based on the consensus of more than two psychiatric doctors, i.e., suicide attempt, agitation or insomnia, psychiatric history (past psychiatric hospitalization or treatment), and anxiety/depression, among others.

Primary psychiatric diagnoses for those who received consultation were also confirmed based on the consensus of more than two psychiatrists. We referred to not only medical records, but also diagnostic processes at psychiatric department liaison-consultation team conferences, which are held once a week. Psychiatric diagnoses for all who receive a consultation are confirmed at the conference after discussions. In this study, psychiatric diagnoses were categorized according to the International Classification of Disease and Infirmity (ICD-10).

We calculated the $95 \%$ confidence intervals of each cell of the contingency table for statistics. Statistical analysis were performed with EZR (Saitama Medical Center, Jichi Medical University), which is a graphical user interface for $\mathrm{R}$ (The $\mathrm{R}$ Foundation for Statistical Computing, version 2.13.0).

This study was approved by the Institutional Review Board of Sapporo Medical University (IRB No. 272-40).

\section{RESULTS}

In total, 3293 patients were admitted to the ACCEC at Sapporo Medical University between January 2012 and December 2015; 1578 of these individuals were confirmed dead after transportation to the ACCEC. Of all living 1715 pa-

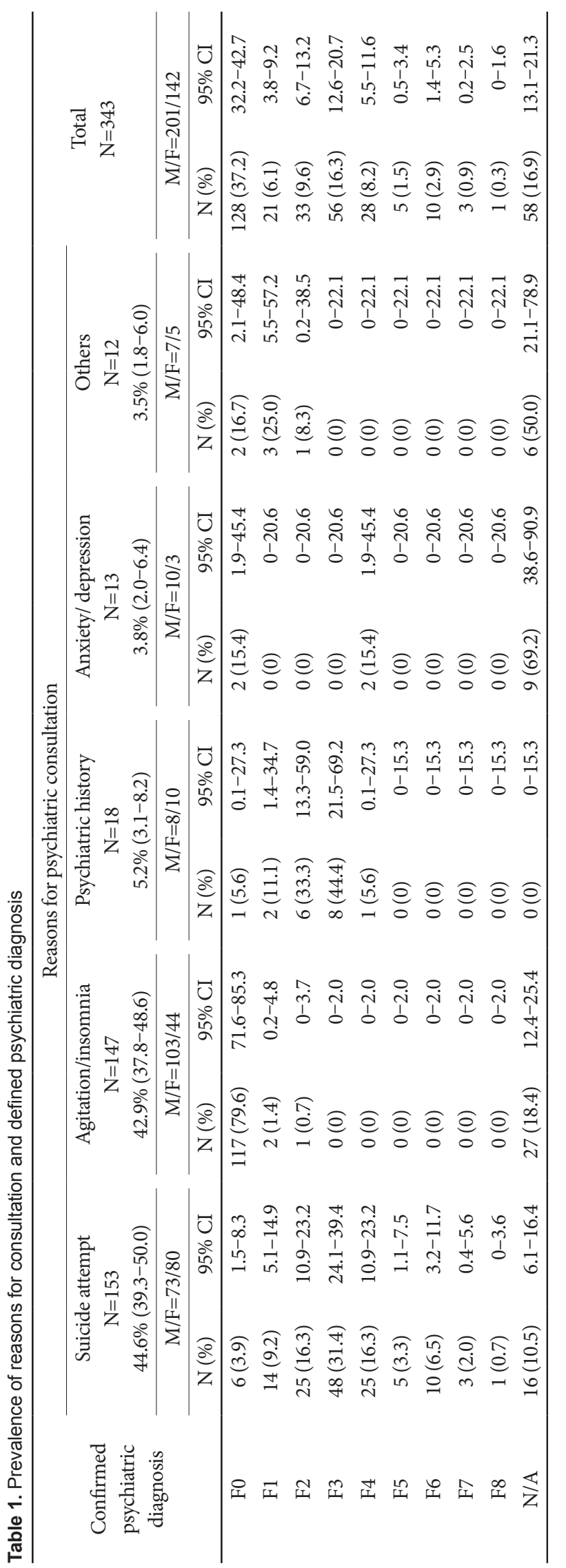


tients, 343 (20\%) received consultations at the psychiatric department after referral from the ACCEC. Of those who were consulted, 201 were men and 142 were women, and the mean age was 56.3 years. The average number days of hospital stay was 22.5 , and the average number of days from admission to consultation to psychiatric department was 5. All participants were Japanese.

The other results are shown in Table 1. The most common reason for consultation was suicide attempt $(n=153,44.6 \%)$ followed by agitation or insomnia ( $n=147,42.9 \%)$, psychiatric history $(n=18,5.2 \%)$, anxiety/depression $(n=13,3.8 \%)$, and others $(n=12,3.5 \%)$. The reasons that were classified as "others" included the prevention of alcohol withdrawal syndrome, and suspected neuroleptic malignant syndrome.

Of all 343 patients, 286 (83.4\%) could be diagnosed with any psychiatric disorders. The remaining $16.6 \%$ could not be diagnosed owing to lack of information due to consciousness disturbance, death, or expedited transport to other hospitals. Organic mental disorder (F0) was the most frequent of all diagnoses according to the ICD-10. The mean age of those who received a diagnosis of organic mental disorder (68.6) was higher than that for all other F-code groups (data not shown).

In the suicide attempt group, of 153 patients, 137 (89.5\%) received psychiatric diagnoses. The most common diagnosis was mood disorder (F3, 31.4\%), followed by schizophrenia (F2, 16.3\%) and neurotic, stress-related and somatoform disorder (F4, 16.3\%) according to the ICD-10.

In the agitation/ insomnia group, of 147 patients, 117 (79.6\%) were diagnosed with organic mental disorder. Delirium accounted for $90.0 \%$ of all patients who were diagnosed with organic disorder, and their mean age was 69.7 years (data not shown).

\section{DISCUSSION}

In the present study, we first revealed a picture of psychiatric consultation from special ED referrals at a metropolitan university hospital in the largest city in northern Japan.

The prevalence of psychiatric consultation was $20 \%$ of all living patients at our ED in the present study, which is a larger proportion compared with those in other advanced countries and Japan..$^{1-4}$ One reason might be due to the characteristics of our ED. Our ED is a special ED where all patients are physically severe cases who easily develop delirium. Another reason might be due to the emergency system in urban areas. In Sapporo, most patients with a high risk of mortality with simultaneous mental illness are exclusively sent to one of five general hospitals including our hospital in the region. These five hospitals have both emergency and psychiatric depart- ments with a psychiatric ward. Another reason might be due to the close relationship between staff at the psychiatry department and our ED. Both departments exist in the same hospital building, and psychiatrists attend regular staff meetings at the ACCEC. This might make it easier for ED staff to refer patients for consultations at the psychiatry department.

The most frequent reason for consultation at our ED was suicide attempt, comprising $44.6 \%$ of all consultations, which was relatively high compared with those in previous studies. One epidemiological study estimated that mental health-related attendances that were due to a suicide attempt or suicidal ideation was $9 \%$, and those due to self-harm was approximately $27 \%$ in Australia and the United Kingdom. ${ }^{3}$ We speculate that suicide attempt, considered the most serious event in mental health, leads ED staff to more actively refer patients to the psychiatric department for consultations.

The most common diagnosis of the reason for consultation regarding agitation/ insomnia was organic mental disorders (79.6\%). In our study, the largest psychiatric assessment for the patients was delirium. One of the most common risk factors of delirium was older age. ${ }^{10}$ The proportion of elderly transported to our ED was $56.6 \%$. Of all ambulance transports in Japan, 56.7\% were elderly, while in the United States, $38.0 \%$ were elderly. ${ }^{11}$ Early diagnosis and management for organic mental disorders including delirium are considered to be important at ED.

Our study had several limitations. First, we did not obtain demographic and clinical data of deceased patients; thus we could not completely count suicide and mental health-related events in the entire targeted population. According to the Japan Trauma Data's annual report, the proportion of registered self-inflicted patients was $5.9 \%$ and the proportion of death by self-inflicted behaviors was $17.7 \%{ }^{12}$ That is, many deaths by suicide might have been included among those who died after transportation to our ED. Second, because we did not have a guideline for psychiatric consultation, the necessity of psychiatric intervention might have been underestimated among ED staff. Furthermore, the demand for psychiatric intervention was likely underrepresented. Another limitation is that our study was based on only one ED in Japan. Therefore, we cannot generalize our findings to other EDs in Japan.

Our study indicated that the frequency of psychiatric consultation was high. This indicates the high demand for mental health services at the ED. A thorough psychiatric assessment can provide adequate psychiatric services to acute patients; thereby possibly preventing suicide attempters from later actually dying by suicide. Based on the results, we suggest educating emergency staff on the initial assessment and care for patients with mental disorders. 
Nevertheless, the data from a single ED cannot be taken as being representative of other EDs in Japan. Further studies investigating multiple facilities are needed.

\section{Acknowledgments}

This work was supported in part by Research and Development Grant for Comprehensive Research for Persons with Disabilities by the Japan Agency for Medical Research and Development [grant number 16768669].

\section{REFERENCES}

1. Larkin GL, Claassen CA, Emond JA, Pelletier AJ, Camargo CA. Trends in U.S. emergency department visits for mental health conditions, 1992 to 2001. Psychiatr Serv 2005;56:671-677.

2. Tankel AS, Di Palma MJ, Kramer KM, van der Zwan R. Increasing impact of mental health presentations on New South Wales public hospital emergency departments 1999-2006. Emerg Med Australas 2011;23: 689-696.

3. Barratt H, Rojas-García A, Clarke K, Moore A, Whittington C, Stockton $\mathrm{S}$, et al. Epidemiology of mental health attendances at emergency departments: systematic review and meta-analysis. PLoS One 2015;11: e0154449.

4. Fire and Disaster Management Agency. Available at: http://www.fdma. go.jp/. Accessed March 18, 2018.
5. Nishi D, Matsuoka Y, Kawase E, Nakajima S, Kim Y. Mental health service requirements in a Japanese medical centre emergency department. Emerg Med J 2006;23:468-469.

6. Downey LV, Zun LS, Burke T. Undiagnosed mental illness in the emergency department. J Emerg Med 2012;43:876-882.

7. Larkin GL, Beautrais AL, Spirito A, Kirrane BM, Lippmann MJ, Milzman DP. Mental health and emergency medicine: a research agenda. Acad Emerg Med 2009;16:1110-1119.

8. Hickey L, Hawton K, Fagg J, Weitzel H. Deliberate self-harm patients who leave the accident and emergency department without a psychiatric assessment: a neglected population at risk of suicide. J Psychosom Res 2001;50:87-93.

9. Kawashima Y, Yonemoto N, Inagaki M, Yamada M. Prevalence of suicide attempters in emergency departments in Japan: a systematic review and meta-analysis. J Affect Disord 2014;163:33-39.

10. Ahmed S, Leurent B, Sampson EL. Risk factors for incident delirium among older people in acute hospital medical units: a systematic review and meta-analysis. Age Ageing 2014;43:326-333.

11. Weiss SJ, Ernst AA, Phillips J, Hill B. Visit to home environments by emergency medical services: a statewide study. Prehosp Emerg Care 2001;5:19-22.

12. Japan Trauma Data Bank Report 2015 (2010-2014). Available at: https://www.jtcr-jatec.org/traumabank/dataroom/data/JTDB2015e. pdf. Accessed March 18, 2018. 